\title{
Effets de la pâture sur la dynamique de la végétation herbacée au Sahel (Gourma, Mali) : une approche par modélisation
}

\author{
Mamadou Oumar Diawara ${ }^{1, *}$, Pierre Hiernaux $^{2}$, Eric Mougin $^{2}$, Manuela Grippa ${ }^{2}$, Claire Delon $^{3}$ \\ et Hawa Salif Diakité ${ }^{4}$ \\ ${ }^{1}$ Département de Biologie, Faculté des Sciences et Techniques (FST), Université des Sciences, des Techniques et des Technologies \\ de Bamako (USTTB), Colline de Badalabougou, B.P. 3206 Bamako, Mali \\ ${ }^{2}$ Géosciences Environnement Toulouse (GET), Observatoire Midi-Pyrénées, Université de Toulouse, CNRS, IRD, 14 avenue Édouard \\ Belin, 31400 Toulouse cedex, France \\ ${ }^{3}$ Laboratoire d'Aérologie (LA), Observatoire Midi-Pyrénées, Université de Toulouse, CNRS, IRD, 14 avenue Édouard Belin, 31400 \\ Toulouse cedex, France \\ ${ }^{4}$ Institut d'Economie Rurale (IER), Centre Régional de Recherche Agronomique de Sotuba (CRRA), Laboratoire Sol-Eau-Plante, \\ Unité Système d'Information Géographique et Télédétection, B.P. 262 Bamako, Mali
}

\begin{abstract}
Résumé - Au Sahel, la végétation se compose d'une strate herbacée principalement composée de plantes annuelles et d'une strate ligneuse peu dense dominée par des épineux. Dans cette région, l'essentiel de la production fourragère a lieu entre juillet et septembre. Le disponible fourrager constitué au cours de cette courte période décroît durant les neuf mois de la saison sèche sous l'effet de plusieurs facteurs, dont la pâture des ruminants domestiques. Les effets de la pâture sur la dynamique saisonnière de la phytomasse herbacée ont fait l'objet de peu d'études au Sahel. La dynamique de saison sèche de la phytomasse herbacée sous l'effet de la pâture est simulée à l'aide du module «Patsec» du modèle STEP. Une étude de sensibilité du module Patsec à la composition spécifique, l'intensité et la saisonnalité de la charge compare les sorties du module aux observations faites en 2010-2011 sur trois sites pastoraux proches de Hombori à 1'Est du Sahel malien. Les résultats montrent que le prélèvement fourrager par le bétail ne contribue que de façon secondaire à la « dégradation » saisonnière de la phytomasse herbacée. Par contre, les effets de la pâture sur le transfert des pailles dressées à la litière et l'enfouissement dans le sol de cette dernière sont nets. L'étude montre également qu'à charge égale exprimée en poids vif, la pâture par les bovins contribue moins au transfert paille-litière et à l'enfouissement de la litière que la pâture de petits ruminants, malgré l'importance du fourrage ligneux dans la ration des chèvres. Le modèle testé reproduit de façon satisfaisante la dynamique saisonnière de la phytomasse herbacée et peut servir d'outil de prévention des crises pastorales liées aux pénuries de fourrage en région sahélienne à travers un suivi de la charge animale et du disponible fourrager de saison sèche.
\end{abstract}

Mots clés : parcours / charge animale / saison sèche / dynamique de la phytomasse / modèle STEP / Sahel

Abstract - Effects of livestock grazing on the dynamics of herbaceous vegetation in Sahel rangeland (Gourma, Mali): a modeling approach. The vegetation of Sahel rangelands is composed of an herbaceous layer dominated by annual plants and a sparse woody plant population dominated by spiny species. Most of the forage production of these rangelands occurs between July and September. The fodder resource available during this short period decreases during the nine months of the dry season as a result of several factors, including grazing by domestic ruminants. The effects of grazing on the seasonal dynamics of the herbaceous mass have been seldom studied. The dry season dynamics of the herbaceous mass under grazing is simulated by the "Patsec" module of the STEP model. In a sensivity analysis of Patsec to grazing seasonality, intensity and to the species composition of grazing herds, the module outputs are compared to the observations made in 2010-2011 in three pastorals sites near Hombori, in Eastern Mali. The results show that the fodder intake by grazing livestock contributes little to the seasonal degradation of the herbaceous mass. However, grazing is determinant to transfer standing herbaceous mass into litter, and to bury the litter in the soil. The study also

\footnotetext{
*Auteur de correspondance : diaprod@hotmail.com
} 
shows that, at equal stocking rate expressed in live weight units, cattle's grazing contributes less to the transfer of standing mass to litter and to the burial of litter than does sheep or goat grazing, despite the large fraction that browse may have in their diet. Patsec and more generally the STEP model are successful in predicting the seasonal dynamics of the rangeland fodder resources. They are a potential tool to prevent the pastoral crisis due to fodder shortage in the Sahelian rangelands by providing seasonal monitoring of the livestock stocking rates and the fodder resource available for grazing.

Keywords: rangelands / stocking rates / dry season / dynamics of phytomass / STEP model / Sahel

\section{Introduction}

Au Sahel, la végétation se compose d'une strate herbacée principalement composée de thérophytes (Cenchrus biflorus, Zornia glochidiata, Alysicarpus ovalifolius, Dactyloctenium aegyptiaca) et d'une strate ligneuse très éparse, dominée par des épineux (Hiernaux et Le Houérou, 2006). La pousse de la strate herbacée a lieu avec les premières pluies de mousson et les jeunes plantules se développent rapidement entre mi-juillet et fin septembre (Grouzis, 1992). Ce développement est contrôlé par l'eau disponible dans le sol, mais aussi par la disponibilité en éléments minéraux comme l'azote et le phosphore (Penning de Vries et Djiteye, 1982).

Après cette courte période de vie active, la végétation se dessèche sur pied et constitue le disponible fourrager pour le bétail durant les 9 à 10 mois de la saison sèche (Boudet, 1987). Érigée ou en litière, cette masse herbacée décroît tout au long de la saison sèche, même en absence de pâture (Grouzis, 1987; Boudet, 1989). Une partie de cette perte est liée à la dispersion des semences et, pour certaines dicotylédones comme Zornia glochidiata, Tribulus terrestris, Alysicarpus ovalifolius, à la chute des feuilles en début de saison sèche (Hiernaux et al., 1994) et aux effets du climat (pluies, humidité relative de l'air). Les pertes supplémentaires sont dues aux insectes phytophages, aux rongeurs, mais aussi à la décomposition microbienne et fongique liée à l'humidité atmosphérique (Hiernaux et al., 2013).

Toutefois, le rôle du bétail dans ces processus est déterminant car il accélère la disparition de la masse herbacée à travers le prélèvement fourrager (Guérin et al., 1987) et participe par le piétinement à la conversion d'une partie de la paille dressée en litière tout en contribuant à l'enfouissement de la litière au sol (Boudet, 1989). Les variations spatiotemporelles de ces pertes de masse herbacée sur pied au cours de la saison sèche sont liées aux différences de composition des rations alimentaires des espèces animales (Guérin et al., 1987), à l'hétérogénéité de la composition botanique des parcours et à la variété des modes d'utilisation des ressources fourragères.

Par ailleurs, la masse herbacée n'est pas la seule ressource fourragère disponible pour le bétail en saison sèche (Hiernaux et al., 1994). En effet, les plantes ligneuses, ou tout au moins certaines d'entre elles (Pilostigma reticulatum, Combretum glutinosum), gardent leurs feuilles pendant de longs mois au cours de cette période. Au cours de la deuxième moitié de la saison sèche, le fourrage aérien ainsi que les gousses d'Acacia sp constituent une ressource d'appoint pour le bétail (Zampaligré, 2012). Pour les petits ruminants et particulièrement les caprins, cette ressource peut représenter jusqu'à $85 \%$ de la composition de la ration (Guérin et al., 1989).

Dans les situations pastorales où le parcours est utilisé par plusieurs espèces animales, le prélèvement fourrager est estimé tout au plus au tiers de la masse herbacée disponible en fin de saison des pluies (Breman et de Ridder, 1991). Au Sahel, où l'élevage pastoral représente la principale activité économique (Wane, 2006), il est utile de disposer d'outils de quantification et de suivi de la dynamique saisonnière de la masse herbacée sèche, principale ressource fourragère du bétail.

Cette étude a pour objectif d'évaluer la sensibilité du module Patsec du modèle STEP (Sahelian Transpiration Evaporation and Production model), à la saisonnalité et à la composition spécifique de la charge animale en saison sèche. Le module Patsec simule la dynamique des pailles et litières, et le comportement fourrager des ruminants au cours de la saison sèche. Il est intégré au modèle STEP dont le module principal simule la production herbacée en saison des pluies (Mougin et al., 1995; Tracol et al., 2006). L'analyse de sensibilité est faite à partir de scénarios dont les simulations sont comparées aux situations observées pendant la saison sèche 2010-2011 sur trois sites pastoraux situés près de Hombori, dans le Gourma au Mali.

\section{Matériel et méthodes}

\subsection{Sites de l'étude}

Les sites de mesure de végétation utilisés dans cette étude ont été mis en place par l'actuel ILRI (International Livestock Research Institute) et l'Institut d'économie rurale (IER) en 1984, dans le cadre d'un projet chargé d'évaluer l'impact de la sècheresse de 1983-1984 sur un système d'élevage pastoral dans le Gourma. Dans le cadre de ce projet piloté par Hiernaux et Diarra, un suivi de la dynamique de la végétation naturelle a été effectué de 1984 à 1993 (Hiernaux et Diarra, 1993). Les observations ont été reprises dans les années 2000 et depuis 2004, elles sont assurées par les équipes du service d'observation AMMA-CATCH (Analyses multidisciplinaires de la mousson africaine-Couplage de l'atmosphère tropicale et du cycle hydrologique). Depuis la crise sécuritaire du nordMali, le suivi se limite à 5 sites pastoraux répartis à proximité du village de Hombori (Fig. 1).

Parmi les 5 sites suivis, 3 ont été retenus pour cette étude, à la fois pour la disponibilité des données de pluies journalières et de charge animale indispensables aux simulations. Il s'agit des sites d'Agoufou $\left(15,34^{\circ} \mathrm{N}, 1,48^{\circ} \mathrm{W}\right)$, de Timbadior $\left(15,34^{\circ} \mathrm{N}, 1,55^{\circ} \mathrm{W}\right)$ et de $\operatorname{Tara}\left(15,23^{\circ} \mathrm{N}, 1,58^{\circ} \mathrm{W}\right)$ situés près de points d'eau permanents.

\subsection{Description et fonctionnement du module Patsec}

Le modèle STEP simule la production et la dynamique de la masse herbacée au Sahel (Mougin et al., 1995). Le modèle 


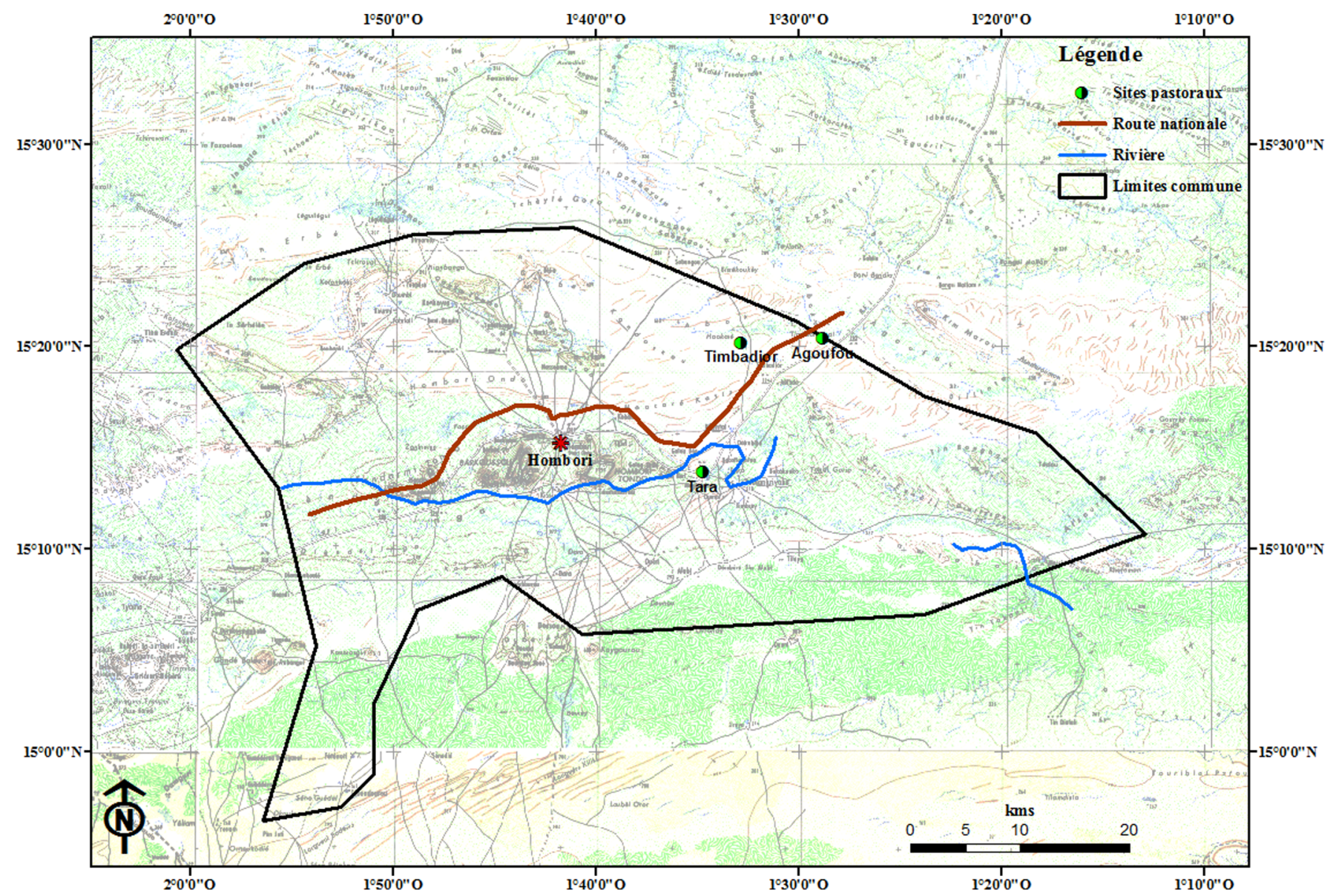

Fig. 1. Localisation des sites de mesures de végétation du Service d'observations AMMA-CATCH au Mali.

Fig. 1. Location of vegetation monitoring sites of the AMMA-CATCH Observations Service in Mali.

comporte deux modules principaux simulant d'une part la production primaire et d'autre part la dégradation de la masse herbacée sur pied au cours de la saison sèche (module Patsec). Le code est écrit en langage Fortran. Le modèle tourne à un pas de temps journalier et nécessite, en entrée, des variables météorologiques (précipitations, température et humidité relative de l'air, rayonnement global, vitesse du vent), de la composition végétale (proportion de plantes à cycle en $\mathrm{C} 3$ et en $\mathrm{C} 4$, proportion de monocotylédones et dicotylédones) et des caractéristiques du sol (pente, profondeur et texture) et de la charge animale. Les paramètres du module Patsec sont présentés dans le tableau 1.

Aux facteurs intrinsèques de dégradation de la masse herbacée sèche sur pied s'ajoutent les feux de brousse qui peuvent causer des pertes considérables. La figure 2 présente schématiquement le processus de dégradation de la masse herbacée au cours de la saison sèche.

La dispersion des semences intervient après la fructification des herbacées annuelles, lorsque la pluviométrie annuelle et la photopériode permettent à celles-ci de boucler leur cycle végétatif. Des travaux de Hiernaux et al. (1994) ont permis d'identifier six stratégies de dispersion de ces diaspores en fonction de la date et de la durée de la période de chute: pendant la saison des pluies, très précoce, précoce, progressive, tardive et très tardive. Elle est représentée par une
Tableau 1. Principaux paramètres du module Patsec et références associées.

Table 1. Parameters of the Patsec module and the associated references.

\begin{tabular}{lll}
\hline Paramètres & Valeurs & Sources \\
\hline $\begin{array}{l}\text { Biomasse initiale } \\
\text { Proportion des plantes }\end{array}$ & $1 \mathrm{~g} \mathrm{MS} / \mathrm{m}^{2}$ & $\begin{array}{l}\text { Mougin } \text { et al., } 1995 \\
\text { à cycle en C3 et en C4 }\end{array}$ \\
$\begin{array}{ll}\text { Ingestion fourragère } \\
\text { 9 } 57 \%\end{array}$ & Hiernaux et al., 2009 \\
& $\mathrm{kg} \mathrm{PV} /$ & Boudet, 1968 \\
\hline
\end{tabular}

fonction exponentielle utilisée pour les six types de dispersions, mais avec cinq constantes spécifiques $(\mathrm{a}, \mathrm{b}, \mathrm{c}, \mathrm{d}$ et e). Ces fonctions sont ensuite pondérées par la contribution relative de chaque mode de dispersion, déterminée à partir de la composition floristique de la strate herbacée. Des valeurs par défaut pour chaque mode peuvent être utilisées en l'absence de mesures de composition floristique, en s'appuyant sur la contribution des dicotylédones (Dicot) et des monocotylédones (1-Dicot) à la formation de la strate herbacée, respectivement : $0,2,0,2,0,2 *(2-D i c o t), 0,2,0,1 *$ (Dicot). Au Sahel, le mode de dispersion le plus rencontré est le mode précoce (Tracol, 2004) 


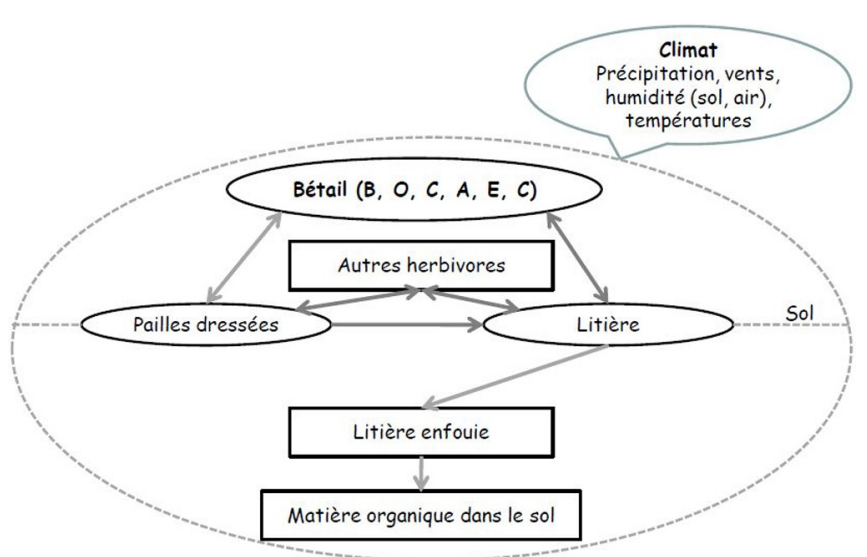

Fig. 2. Représentation schématique du module de dégradation de la masse herbacée sèche. Les lettres $\mathrm{B}, \mathrm{O}, \mathrm{C}, \mathrm{A}, \mathrm{E}, \mathrm{C}$ désignent respectivement bovin, ovin, caprin, asin, équin et camelin (adaptée de Hiernaux et al., 2013).

Fig. 2. Schematic representation of dry season herbaceous mass degradation module. The letters $B, O, C, A, E, C$ stand for bovine, ovine, goat, asin, equine and camel (adapted from Hiernaux et al., 2013).

et cette dispersion est formalisée par l'équation suivante:

$$
\operatorname{Disp}(i)=a+b\left(\frac{\text { ifruct }}{3}\right) c . e^{\left(d+f\left(\frac{\text { ifruct }}{s}\right)\right)},
$$

où Disp (i) est la fonction de dispersion des diaspores de type $I$ et Ifruct, le nombre de jours écoulés depuis la date de fructification.

Le climat joue un rôle important dans la dégradation saisonnière de la masse herbacée au Sahel (Delon et al., 2015). Dans cette région où il fait chaud et parfois humide, l'humidité du sol, mais aussi l'humidité de l'air, participent à la décomposition de la litière au sol et à la minéralisation de la litière enfouie, en particulier sous l'action des termites. D'autres facteurs climatiques, comme le vent, favorisent la dispersion des semences et la chute des feuilles de certaines dicotylédones comme Zornia glochidiata, Alysicarpus ovalifolius et Tribulus terrestris. L'effet du climat sur la disparition de la masse herbacée au cours de la saison sèche est décrit par l'équation:

$$
K n=\operatorname{Lit}_{\max } \cdot \sqrt{f(\text { hum }) . f(\text { precip })} . f(\text { temp }) . f(\text { wind }),
$$

où $K n$ représente le taux de disparition de la masse herbacée (principalement la litière) dû au climat. Les effets de l'humidité de l'air (f(hum)), des précipitations (f(precip)), du vent $(f($ wind $))$ et de la température moyenne $(f($ temp $))$ sont pris en compte dans le calcul de $K n$. Dans cette équation, Lit $_{\max }$ représente le taux maximum de litière qui dépend de la proportion de monocotylédones et dicotylédones.

L'ingestion par le bétail est modulée en fonction de la disponibilité des ressources et de la préférence alimentaire de l'espèce animale concernée. Pour les caprins et les camelins, le fourrage aérien (feuilles + gousses) peut représenter jusqu'à $85 \%$ de la ration quotidienne alors que pour les bovins, les asins et les équins, c'est le fourrage herbacé qui domine.

L'ingestion désignée par Kig dépend de la quantité maximale ingérable par le bétail IngMaxB ( $\mathrm{g}$ de matière sèche, MS), fonction de la charge animale $L V s t k$ (UBT/ha) et de la disponibilité de la ressource $\left(\mathrm{g} \mathrm{MS} / \mathrm{m}^{2}\right)$. Les taux de charge animale en poids métabolique $K c h$ (UBT métabolique/ha) et la quantité maximale ingérable par le bétail $\left(\mathrm{g} \mathrm{MS} / \mathrm{kg} \mathrm{PV}^{0,75}\right.$, jour) sont calculés par les équations suivantes :

$$
\begin{gathered}
K c h=\sum_{1}^{6} \operatorname{Pmeta}(i) \cdot \operatorname{LV} \operatorname{stk}(i), \\
\operatorname{IngMaxB}=\sum_{1}^{6} \operatorname{IngMax}(i) \cdot \operatorname{LV} \operatorname{stk}(i) .
\end{gathered}
$$

Dans l'équation de la charge animale, Pmeta représente un coefficient spécifique à l'espèce animale (bovin, ovin, caprin, asin, équin et camelin) et qui permet d'exprimer la charge animale en poids métabolique. L'ingestion $\mathrm{Kig}$ est donc formalisée de la manière suivante:

$$
\text { Kig }=\frac{\operatorname{IngMaxB}}{\text { Bstd }} .
$$

Dans cette formule, Bstd représente la masse de paille dressée $\left(\mathrm{g} \mathrm{MS} / \mathrm{m}^{2}\right)$ et $\operatorname{IngMaxB}$, la quantité maximum de fourrage ingérable par le bétail. Le taux de transfert de la paille en litière dû au piétinement est désigné par $K t$ et décrit par l'équation suivante :

$$
\begin{gathered}
K t=K c h M L V s t k \\
M L V s t k=0,2 e^{(-0,00068 \text { Bstd }(1-\text { Dicot }))} \\
+0,165 e^{-0,00092 B s t d D i c o t}
\end{gathered}
$$

MLVstk représente l'effet du piétinement sur les monocotylédones et les dicotylédones.

\subsection{Estimation des phytomasses herbacées}

L'estimation des phytomasses herbacées est réalisée tous les 10 jours pendant la saison des pluies et une fois par mois pendant la saison sèche le long de transects de $1000 \mathrm{~m}$ sur les sites pastoraux. Le long de ces transects, la strate herbacée est échantillonnée et décrite visuellement mètre par mètre de façon à estimer la surface relative des classes de densité de végétation dans une série linéaire de 1000 quadrats. Les quadrats sont répartis entre quatre classes de densité de végétation herbacée (plages de sol $n u=O$, densité basse $=B$, médiane $=\mathrm{M}$ et haute $=\mathrm{H}$ ). Plusieurs faciès peuvent être échantillonnés en fonction du site, à cause de l'hétérogénéité spatiale du couvert végétal due aux conditions géomorphologiques (système de dunes et d'interdunes). Dans chaque faciès, 3 échantillons de $1 \mathrm{~m} \times 1 \mathrm{~m}$, dont l'emplacement est tiré aléatoirement le long du transect, sont décrits dans les classes basse et haute, et 6 dans la strate moyenne. Des récoltes intégrales, par fauche ou par prélèvement de la litière selon la saison, sont effectuées sur chaque échantillon de $1 \mathrm{~m} \times 1 \mathrm{~m}$ et la masse $\left(\mathrm{g} \mathrm{MS} / \mathrm{m}^{2}\right)$ est déterminée après séchage (Hiernaux et al., 2009). 


\subsection{Estimation de la charge animale}

Les effectifs de bétail et la composition des troupeaux qui ont fréquenté les trois sites pastoraux ont été estimés mensuellement sur le terrain autour des principaux points d'eau pastoraux. Les charges animales (UBT/ha) correspondantes sont estimées à partir de l'exploitation de la ressource fourragère autour de chaque point d'eau. Le rayon d'exploitation de la ressource par le bétail s'étend de 6 à $12 \mathrm{~km}$. Dans cette approche, la distance minimale de $6 \mathrm{~km} /$ jour coïncide avec le mois d'octobre et augmente d'un kilomètre chaque fois que les ressources diminuent de $20 \%$, jusqu'à une distance maximale de $12 \mathrm{~km}$ (Diawara et al., en préparation).

\subsection{Analyses de sensibilité}

Une analyse de sensibilité de la dégradation des pailles et litières aux variations de la charge animale est conduite à partir de scénarios de variation de la charge animale. Les scénarios sont construits à partir des charges animales relevées en 2010 et 2011 dans l'aire de desserte du site d'Agoufou et de ses environs. Les règles choisies pour construire ces scénarios s'appuient sur les variations observées de la charge saisonnière et de la variation de sa composition (proportion de petits ruminants par rapport aux bovins) ou sur la variation du calendrier d'utilisation de la ressource par le bétail.

\subsubsection{Variation dans l'intensité de la pâture}

La construction des scénarios de pâture se fonde sur la mobilité saisonnière du bétail qui entraîne une concentration périodique du bétail autour des points d'eau, provoquant une pénurie de fourrage dans les environs immédiats du point d'eau. Dans le premier scénario, la charge animale maximale de l'aire de desserte de la mare d'Agoufou est modifiée de plus ou moins $10 \%$ des charges observées et dans le second de plus ou moins $50 \%$.

\subsubsection{Variation de la composition de la charge animale}

Les scénarios s'inscrivent dans l'optique d'un changement tendanciel de la composition spécifique du cheptel, tel qu'observé dans différentes localités du Sahel (Manoli, 2012), avec une contribution plus ou moins élevée des petits ruminants. Trois situations sont testées à charge totale égale en poids vif: une première pour laquelle la pression de pâture est exclusivement bovine, une seconde pour laquelle elle repose exclusivement sur les petits ruminants et une troisième pour laquelle les bovins représentent la moitié de la charge et les petits ruminants l'autre moitié; proportion observée en moyenne sur la saison sèche 2010-2011.

\subsubsection{Calendrier d'utilisation de la ressource}

Trois scénarios sont imaginés pour évaluer les effets d'une pâture de saison sèche différée sur la dynamique saisonnière de la ressource, à charge égale en poids vif. Le premier scénario est basé sur l'absence de pâture au début de la saison sèche (octobre-novembre), le second sur l'absence de pâture en décembre-janvier et le troisième sur une absence de pâture durant les quatre premiers mois de la saison sèche, soit d'octobre à janvier.

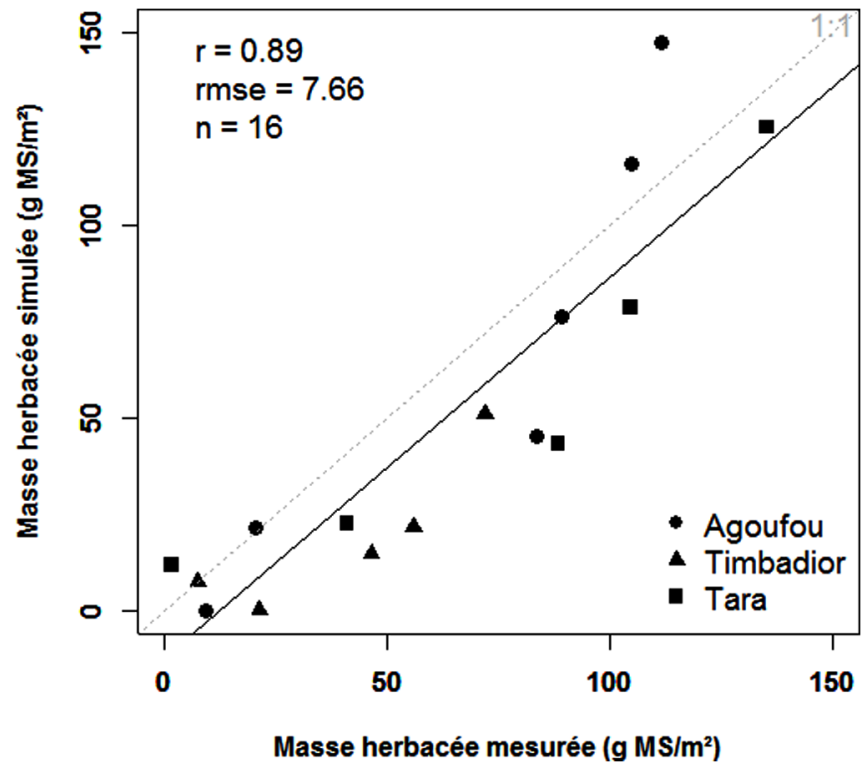

Fig. 3. Relation entre les masses herbacées mesurées et simulées.

Fig. 3. Relationship between measured and simulated herbaceous masses.

\section{Résultats}

\subsection{Comparaison des masses herbacées mesurées et simulées}

Les résultats de simulation de la dynamique des masses herbacées sont comparés aux mesures faites sur les trois sites pastoraux au cours de la saison sèche 2010-2011 (Fig. 3). La relation déterminée par un coefficient de corrélation $(\mathrm{r}=0,89)$ montre que Patsec arrive à bien reproduire la dynamique de la phytomasse herbacée pendant la saison sèche. La légère sousestimation des valeurs de phytomasse simulées peut être expliquée par des charges animales inférieures à la pression exercée réellement sur les parcours.

\subsection{Sensibilité de la dégradation saisonnière de la masse herbacée à la variation de la charge animale}

L'impact des variations de la charge animale sur le processus de dégradation des pailles et des litières a été évalué sur le site d'Agoufou (Fig. 4). Une baisse de $50 \%$ de la charge moyenne, estimée sur le site pastoral d'Agoufou en 2010-2011 $(0,08 \mathrm{UBT} / \mathrm{ha})$, prolonge de 50 à 60 jours la période d'utilisation potentielle de la paille dressée, mais cette baisse a une faible incidence sur le transfert de la paille dressée en litière. Par contre, une variation de plus ou moins $10 \%$ de cette charge n'a pas d'effets sur le transfert et a peu d'effet sur le calendrier de transfert des pailles.

L'influence du calendrier de pâture sur la dynamique de la ressource fourragère au cours de la saison sèche est testée pour trois scénarios comparés au calendrier observé en 2010-2011, à charge égale sur la saison (Fig. 5). Dans la situation observée, ainsi que dans les trois scénarios, Patsec simule des quantités de paille dressée atteignant $0,16 \mathrm{~g} \mathrm{MS} / \mathrm{m}^{2}$ en fin de saison sèche. Par contre, une mise en défens, même de courte durée, permet de conserver au sol une litière plus abondante. Pour le 

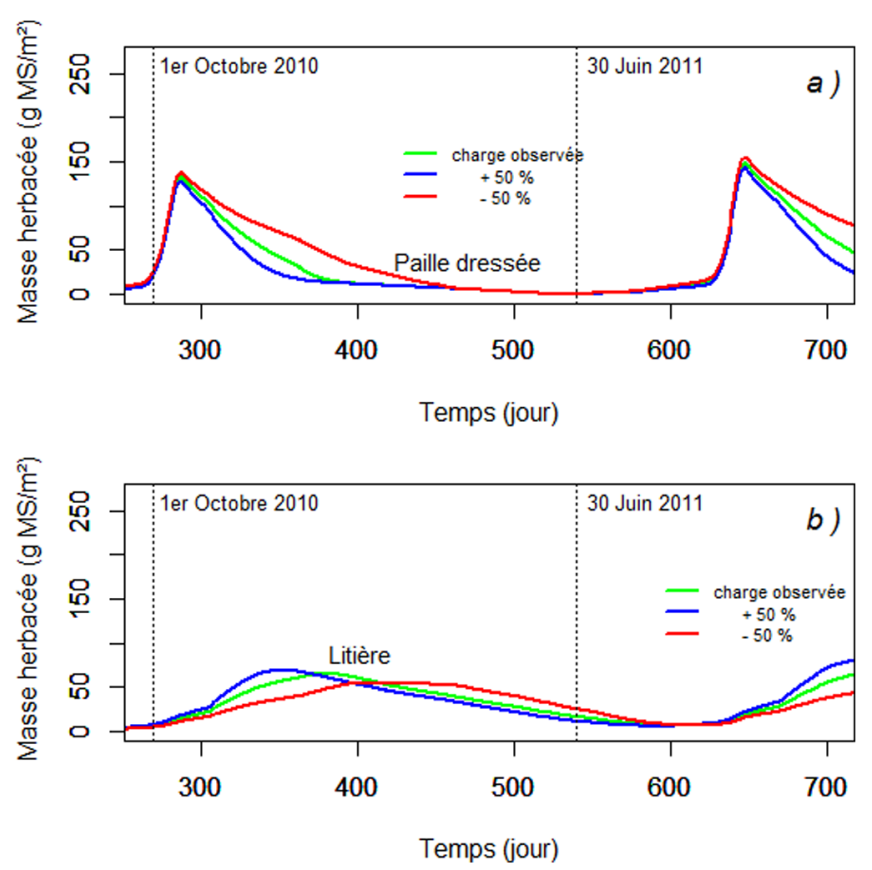

Fig. 4. Dynamique de la masse herbacée en fonction de la variation de la charge animale ( $\pm 50 \%$ de la charge animale estimée) dans la zone de desserte de la mare d'Agoufou. a) pailles dressées; b) litière.

Fig. 4. Dynamics of the herbaceous mass as a function of the variation of the stocking rate ( $\pm 50 \%$ of the observed stocking rate) in the service area of Agoufou pond. a) standing straws; b) litter.

scénario 3 (mise en défens première moitié) par exemple, le modèle simule une litière au sol de $29,11 \mathrm{~g} \mathrm{MS} / \mathrm{m}^{2}$ en fin de saison sèche, contre $15,02 \mathrm{~g} \mathrm{MS} / \mathrm{m}^{2}$ pour la situation observée.

La dynamique de la phytomasse herbacée sèche en fonction de la variation de composition spécifique de la charge animale est testée pour trois scénarios (Fig. 6). À charge égale exprimée en poids vif, les petits ruminants ingèrent plus de fourrage que les bovins. Une pâture exclusivement par les bovins permet de garder une litière au sol plus importante en fin de saison sèche, qu'une pâture effectuée par des troupeaux mixtes ou par les élevages monospécifiques de petits ruminants.

\section{Discussion}

La dégradation de la masse herbacée n'est pas homogène au cours de la saison sèche et sa variabilité dépend de plusieurs facteurs, dont l'humidité résiduelle due aux pluies tardives comme ce fut le cas en 2010 ou à d'éventuelles pluies précoces d'avant mousson (2011). Cela explique les taux de pertes élevés observés sur le site d'Agoufou en 2010 avec une perte de $23 \mathrm{~g} \mathrm{MS} / \mathrm{m}^{2}(9,9 \%)$ entre le 7 et le 15 octobre, malgré une faible pression pastorale à cette période de l'année $(0,01 \mathrm{UBT} / \mathrm{ha})$. Cette dégradation de début de saison sèche n'est pas exceptionnelle au Sahel. Ces pertes sont proches de celles rapportées par Hiernaux et al. (2013) sur des parcours similaires dans la région du Gourma et de celles rapportées par Grouzis (1987) sur des parcours du Sahel burkinabé.

Cette perte de masse dans les premiers mois peut même atteindre $40 \%$ de la masse initiale (Hiernaux et al., 2013). La
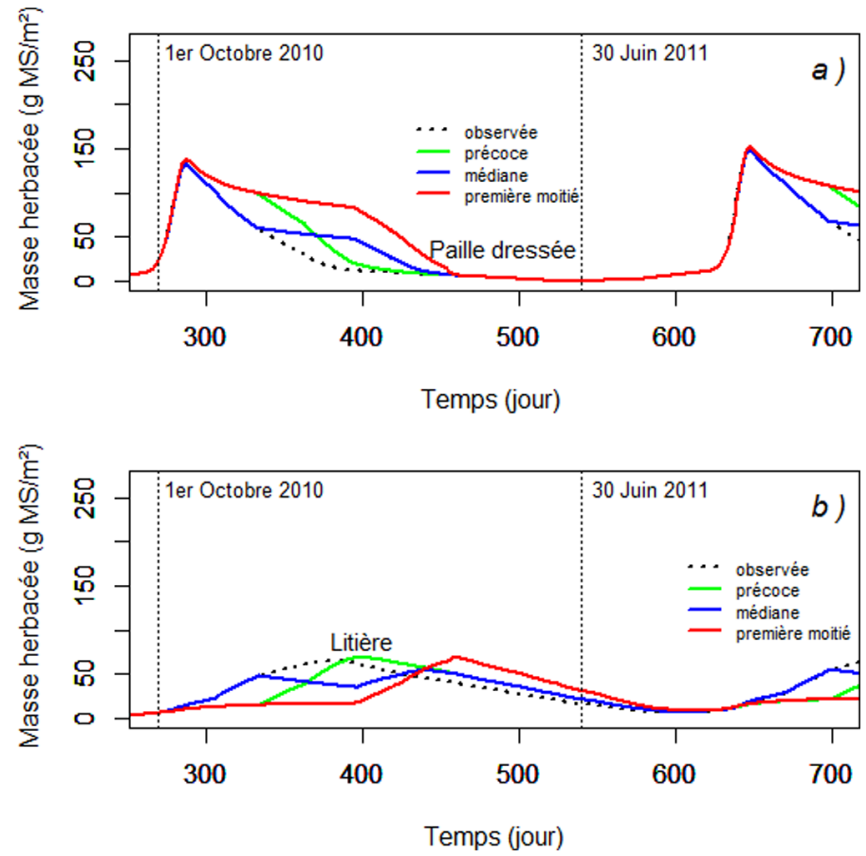

Fig. 5. Dynamique de la masse herbacée sèche en fonction du calendrier de pâture dans la zone de desserte de la mare d'Agoufou. Observation $=$ calendrier observé en 2010 -2011 ; scénario $1=$ mise en défens précoce; scénario $2=$ mise en défens médiane; scénario $3=$ mise en défens première moitié. a) dynamique des pailles dressées; b) dynamique de la litière.

Fig. 5. Dynamics of the dry herbaceous mass according to the grazing calendar near the Agoufou pond. Observation = observed calendar in 2010-2011; pattern 1=early exclosure; pattern 2=mid-season exclosure; pattern $3=$ first half exclosure. a) standing straw; b) litter.

dégradation de fin de saison sèche simulée par le modèle est moins importante que celle du début de la saison sèche. Cette différence est en partie due à la dispersion des semences en début de saison sèche. Cependant, elle est supérieure aux moyennes mensuelles observées en milieu de saison sèche, ce qui peut s'expliquer par la remontée de l'humidité de l'air et des températures qui activent la décomposition organique des litières.

La pâture ne modifie de façon significative la dynamique de la masse herbacée sèche que lorsqu'elle atteint ou dépasse $30 \%$ de la charge moyenne $(0,08 \mathrm{UBT} / \mathrm{ha})$ relevée sur le site d'Agoufou en 2010-2011. Ce résultat rejoint la perception de certains éleveurs rapportée par Boutrais (1994), qui considèrent que les zébus à longues cornes effectuent des parcours quotidiens de faible amplitude et n'ingèrent que de petites quantités de fourrage. À charge animale égale exprimée en poids vif, les petits ruminants ingèrent plus de fourrage que les bovins (Guérin et al., 1987; Ayantunde et al., 2006) et participent davantage au transfert de la paille dressée en litière. La variation de plus (ou moins) $50 \%$ de la charge saisonnière observée sur le site d'Agoufou en 2010 peut écourter (ou rallonger) la disponibilité de la paille dressée, préférée par les bovins qui représentent $83 \%$ de la charge animale (Diawara et al., en préparation).

L'impact de la mise en défens sur la dynamique de la ressource fourragère de saison sèche est net quand elle est mise 

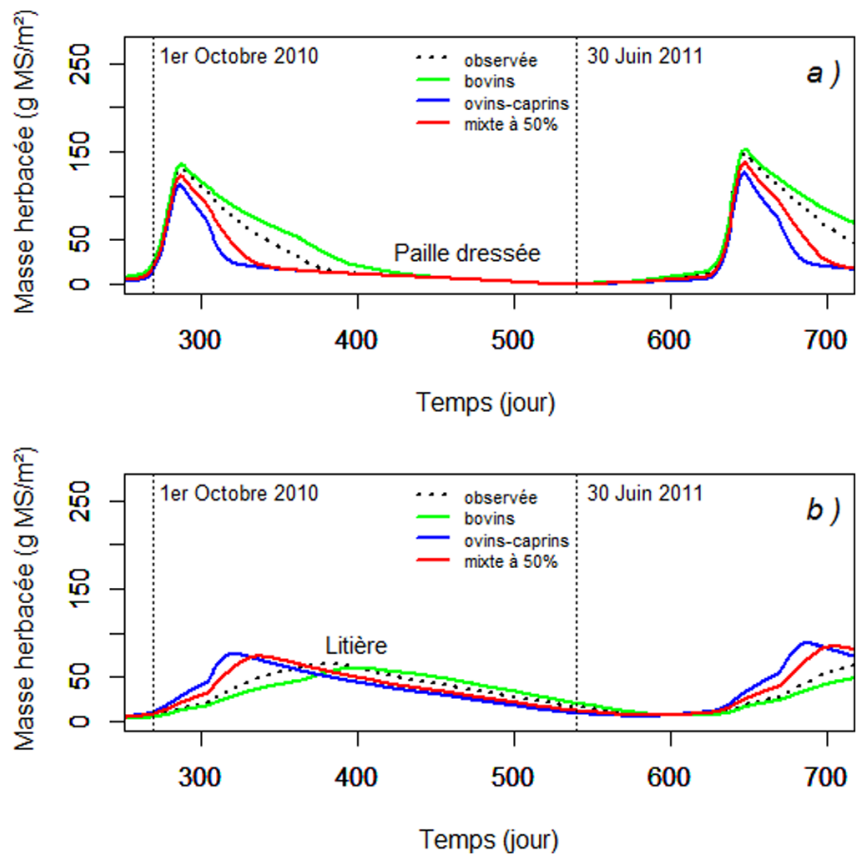

Fig. 6. Dynamique de la masse herbacée sèche en fonction de la composition spécifique des troupeaux dans la zone de desserte de la mare d'Agoufou. Observation= composition moyenne observée en 2010-2011; scénario 1=bovins ; scénario 2=ovins-caprins; scénario $3=$ mixte à $50 \%$ bovins-(ovins-caprins). a) paille dressée; b) litière.

Fig. 6. Dynamics of the dry herbaceous mass according to the specific composition of livestock in the service area of Agoufou pond. Observation = observed composition in 2010-2011; pattern $1=$ exclusively bovine; pattern 2 = stocking rate made up exclusively by small ruminants; pattern $3=50 \%$ of cattle, the other half by small ruminants. a) standing straw; b) litter.

en place après la chute des semences, qui intervient à partir de la fin des pluies en fonction du mode de dispersion des semences. Une mise en défens en fin de saison sèche n'a pas d'effets sur la disponibilité du fourrage herbacé pour le bétail. Par contre, elle permet de maintenir une litière au sol nécessaire à la protection contre l'érosion éolienne et hydrique et à la régénération du parcours à travers le maintien au sol des semences (Boudet, 1989).

$\mathrm{Au}$ Sahel, la plupart des outils d'alerte précoce s'appuient sur une évaluation du disponible fourrager de fin de saison de croissance, sans tenir compte de l'utilisation de ce stock durant la longue saison sèche (SIPSA, 2005). Le modèle STEP avec son module Patsec permet de simuler la variation du disponible fourrager de saison sèche, en fonction de l'utilisation pastorale. À plus grande échelle, il pourrait ainsi contribuer à atténuer, voire prévenir, des crises pastorales, en améliorant l'information quantitative sur les disponibilités fourragères de la longue saison sèche (Boutrais, 1994).

\section{Conclusion}

Le modèle STEP reproduit de façon satisfaisante la dynamique de la masse herbacée tout au long de l'année. Malgré l'échelle réduite et le nombre de sites pastoraux sur lequel les tests ont été effectués, l'étude offre de bonnes perspectives de suivi de la dynamique des écosystèmes pastoraux au Sahel. En fonction de la pluviosité annuelle et de la charge animale estimée localement, le modèle permet de simuler la dynamique de sa phytomasse herbacée, et donc d'anticiper d'éventuelles pénuries de fourrage qui peuvent survenir localement.

À terme, les charges animales estimées autour de points d'eau pastoraux et utilisées comme variable d'entrée pourraient être remplacées par des cartes de charges pour des simulations étendues à des échelles spatiales plus importantes. Cependant, leur établissement nécessite la mise en place d'un réseau d'information participatif.

Le modèle STEP dans son ensemble pourrait être couplé à des modèles existants de gestion de la fertilité des sols. D'autres perspectives de couplage sont à envisager avec des modèles de nutrition et de production animale comme LIVSIM (Rufino et al., 2009), pour évaluer la productivité des troupeaux pastoraux.

Remerciements. Ce travail a vu le jour grâce à un financement accordé par l'Agence nationale de la recherche dans le cadre du projet ECliS (Élevage, climat et société, ANR-AA-VULNS003). Les auteurs remercient les chercheurs, techniciens et enquêteurs de l'Observatoire AMMA-CATCH au Mali. Ils remercient les populations, et en particulier les éleveurs de la commune de Hombori de la qualité de leur accueil.

\section{Références}

Ayantunde A, Fernandez-Rivera S, Hiernaux P, Van Keulen H, Udo HJM. 2006. Day and night grazing by cattle in the Sahel. Journal of Range Management 2: 144-149.

Boudet G. 1968. Emploi pratique des analyses fourragères pour l'appréciation des pâturages tropicaux. REMVT 21: 227-266.

Boudet G. 1987. Connaissance et gestion de l'espace pastoral sahélien, Terroirs pastoraux et agro-pastoraux en zone tropicale. Étude et synthèse de l'IEMVT n ${ }^{\circ}$ 24. Maisons-Alfort: IEMVT, pp. 5-59.

Boudet G. 1989. Évolution de la végétation des parcours sahéliens et possibilités de réhabilitation. Fourrages 120: 401-415.

Boutrais J. 1994. Éleveurs, bétail et environnement. À la croisée des parcours, pasteurs, éleveurs, cultivateurs. Paris: Éditions de l'ORSTOM, pp. 304-319.

Breman H, de Ridder N. 1991. Manuel sur les pâturages des pays sahéliens. Paris, Wageningen (Pays Bas) : A.CrT-cabo-Dio-CTA, Karthala, 485 p.

Delon C, Mougin E, Serça D, Grippa M, Hiernaux P, Diawara M, et al. 2015. Modelling the effect of soil moisture and organic matter degradation on biogenic NO emissions from soils in Sahel rangeland (Mali). Biogeosciences 12: 3253-3272. Doi: 10.5194/ bg-12-3253-2015.

Diawara MO, Hiernaux P, Mougin E, Kergoat L, Soumaguel N. En préparation. Are the Sahel rangelands overgrazed? Lessons from the joint monitoring of rangeland vegetation and stocking rates in the Hombori district (Mali). Rangeland Ecology and Management, RAMA-S-17-00026-1, en révision.

Grouzis M. 1987. Structure, productivité et dynamique des systèmes écologiques Sahéliens. (Mare d'Oursi, Burkina Faso). Collection Études et Thèses. Paris (France) : ORSTOM.

Grouzis M. 1992. Germination et établissement des plantes annuelles sahéliennes. L'aridité : une contrainte au développement. Paris : ORSTOM, 597 p. 
Guérin H, Friot D, Mbaye ND, Fall ST, Richard D, Diop M, et al. 1987. L'ingestion des fourrages des parcours naturels en zone sahélienne: mesures en stabulation et au pâturage. Reproduction Nutrition Développement 27(1B): 197-198.

Guérin H, Friot D, M'Baye ND, Richard D. 1989. Le régime alimentaire des ruminants domestiques sur les pâturages naturels sahéliens et soudano-sahéliens. Revue Sénégalaise des Recherches Agricoles et Halieutiques 2(1): 48-67. http:/intranet.isra.sn/ aurifere/opac css/docnum/IS0004999.pdf.

Hiernaux P, Diarra L. 1993. Dynamique de la végétation des terres de parcours sahéliennes. Un bilan du suivi des sites pastoraux du Gourma en 1992. Doc.progr.1/93. Addis Ababa: ILCA, $46 \mathrm{p}$.

Hiernaux P, Le Houérou HN. 2006. Les parcours du Sahel. Sécheresse 17: $51-71$.

Hiernaux P, Cissé MI, Diarra L, De Leeuw PN. 1994. Fluctuations saisonnières de la feuillaison des arbres et des buissons sahéliens. Conséquences pour la quantification des ressources fourragères. REMVT 47(1): 117-125.

Hiernaux P, Mougin E, Diarra L, Soumaguel N, Lavenu F, Tracol Y, et al. 2009. Sahelian rangeland response to changes in rainfall over two decades in the Gourma region, Mali. Journal of Hydrology 375: 114-117.

Hiernaux P, Mougin E, Diawara M, Soumaguel N, Diarra L. 2013. How much does grazing contribute to herbaceous decay during the dry season in Sahel rangelands? Élevage Climat et Société, ECliS 3.4. Toulouse (France): Géosciences Environnement Toulouse (GET), $22 \mathrm{p}$.

Manoli C. 2012. Le troupeau et les moyens de sécurisation des campements pastoraux : une étude de la gestion des troupeaux de la Communauté rurale de Tessekre, dans le Ferlo sénégalais. Thèse de Doctorat Montpellier SupAgro, 247 p. http://www.supagro.fr/ theses/extranet/12-0032_Manoli.pdf.
Mougin E, Lo Seen D, Rambal S, Gaston A, Hiernaux P. 1995. A Regional Sahelian Grassland Model to be coupled with multispectral satellite data. I: Model description and validation. Remote Sens Environ 52: 181-191.

Penning de Vries FWT, Djiteye MA. 1982. La productivité des pâturages sahéliens. Une étude des sols, des végétations et de l'exploitation de cette ressource naturelle ( $1^{\text {re }}$ édition). Wageningen: PUDOC, $525 \mathrm{p}$.

Rufino MC, Herrero M, Van Wijk MT, Hemerik L, de Ridder N, Giller KE. 2009. Lifetime productivity of dairy cows in smallholder farming systems of the central highlands of Kenya. Animal 3(7): 1044-1056. Doi: 10.1017/S1751731109004248.

SIPSA. 2005. Mise en œuvre d'un système d'information sur le pastoralisme au Sahel. Note introductive préparée par l'Initiative Élevage Environnement et Développement (LEAD) et le Groupement d'intérêt scientifique Pôle Pastoral Zones Sèches (PPZS). http://www.museum.agropolis.fr/pages/savoirs/desertification2/ SIPSA_concept_mai2005.pdf.

Tracol Y. 2004. Étude des variations interannuelles de la production herbacée des pâturages sahéliens: exemple du Gourma Malien, Thèse Doct, SC Université Toulouse III, Paul Sabatier, 270 p. http://www.cesbio.ups-tlse.fr/data all/theses/Th_Tracol_2004.pdf.

Tracol Y, Mougin E, Hiernaux P, Jarlan L. 2006. Testing a Sahelian grassland functioning model against herbage mass measurements. Ecological Modeling 193: 437-446.

Wane A. 2006. Économie du pastoralisme: une analyse bibliographique. Initiative mondiale pour un pastoralisme durable, IUCN EARO, 28 p. https://europa.eu/capacity4dev/file/10091/download? token $=\mathrm{c}-\mathrm{atZq} 8 \mathrm{e}$.

Zampaligré N. 2012. The role of ligneous vegetation for livestock nutrition in the sub-Sahelian and Sudanian zones of West Africa: Potential effects of climate change. Thesis, University of Kassel, 114 p. https://kobra.bibliothek.uni-kassel.de/bitstream/urn:nbn:de: hebis:34-2012120742280/5/ThesisNouhounZampaligre.pdf.

Citation de l'article : Diawara MO, Hiernaux P, Mougin E, Grippa M, Delon C, Diakité HS. 2018. Effets de la pâture sur la dynamique de la végétation herbacée au Sahel (Gourma, Mali) : une approche par modélisation. Cah. Agric. 27: 15010. 